'Departamento de Kinesiología, Facultad de Medicina,

Universidad de Chile. Santiago, Chile.

${ }^{2}$ Facultad de Ciencias de la Salud Blanquerna, Grupo de investigación de Fisioterapia (GReFis), Universidad Ramon Llull. Barcelona, España.

${ }^{3}$ Equipo de Kinesiología, Instituto Nacional del Tórax. Santiago, Chile.

aKinesiólogo. ${ }^{\mathrm{b}} \mathrm{PhD}$.

Los autores declaran no tener conflictos de interés.

Este trabajo no recibió financiamiento.

Recibido el 13 de enero de 2016, aceptado el 9 de marzo de 2017.

Correspondencia a: Roberto Vera-Uribe Av. Independencia 1027, Santiago, Chile. kinerob@yahoo.com

\section{Evaluación de la actividad física en pacientes con enfermedad pulmonar obstructiva crónica}

\author{
RODRIGO TORRES-CASTRO ${ }^{1, a}$, CRISTIAN CÉSPEDES ${ }^{1, a}$, \\ JORDI VILARÓ ${ }^{2, a, b}$, ROBERTO VERA-URIBE ${ }^{1, a}$, \\ MARCELO CANO-CAPPELLACCI ${ }^{1, \mathrm{a}, \mathrm{b}}$, DIEGO VARGAS ${ }^{1,3, \mathrm{a}}$
}

\section{Tools for physical activity assessment in chronic obstructive pulmonary disease}

The prevalence and mortality of chronic obstructive pulmonary disease (COPD) is increasing in Chile, constituting a public health problem. Pulmonary and systemic consequences of COPD affect physical activity, as the disease progresses. There are multiple means for physical activity assessment, from low cost and easily applicable questionnaires to sophisticated laboratory tests. Physical inactivity is a modifiable risk factor for morbidity and mortality in patients with COPD. Physical activity interventions not only contribute to decrease the likelihood of mortality, but also protect from comorbidities, especially cardiovascular ones. It also plays a major role avoiding functional limitations of these subjects. Dyspnea and fatigue render exercise as an unpleasant activity for most patients with COPD. If psychological alterations such as anxiety and depression are summed, these patients drift towards an inactive lifestyle. This article analyzes several tools available to assess physical activity is patients with COPD, useful in clinical practice.

(Rev Med Chile 2017; 145: 1588-1596)

Key words: Exercise; Monitoring, Ambulatory; Monitoring, Physiologic; Pulmonary Disease, Chronic Obstructive.

\section{I} a prevalencia de las enfermedades respiratorias crónicas ha aumentado en los últimos 50 años ${ }^{1}$. Entre estas, destaca la enfermedad pulmonar obstructiva crónica (EPOC). La EPOC se define como una patología prevenible y tratable, parcialmente reversible con el uso de broncodilatadores, caracterizada por una limitación al flujo aéreo persistente, generalmente progresiva y asociada a una respuesta inflamatoria exagerada de las vías aéreas y del parénquima pulmonar frente a partículas de gases nocivos, en especial, el humo de tabaco ${ }^{2}$.

Según la Organización Mundial de la Salud (OMS), se estima que más de 80 millones de personas padecen EPOC de moderada a grave y se proyecta que para el año 2020 la EPOC va a ser la tercera causa de muerte en el mundo 3 . En Chile, la prevalencia reportada en 2005 por el estudio PLATINO fue de $16,9 \%$ en mayores de 40 años.

Las manifestaciones pulmonares y músculo-esqueléticas de la enfermedad alteran la ventilación pulmonar y limitan el aumento de los requerimientos ventilatorios necesarios durante el ejercicio, provocando una mayor sensación de disnea y fatiga ${ }^{5}$. Estos síntomas provocan que el ejercicio no sea una actividad placentera para la mayoría de los pacientes, predisponiéndolos a un estilo de vida inactivo ${ }^{6}$.

Los niveles de actividad física (AF) son marcadamente menores en las personas con EPOC y van disminuyendo de acuerdo a la gravedad de la enfermedad ${ }^{7}$. La literatura reporta que la 
inactividad física es un predictor de mortalidad y de exacerbaciones, contribuyendo a una mayor progresión de la enfermedad ${ }^{8}$. En consecuencia, es necesario determinar el nivel de AF que presenta cada paciente para establecer estrategias terapéuticas adecuadas a las necesidades individuales, evitando el consumo excesivo de recursos sanitarios.

El objetivo de este artículo es revisar las herramientas de evaluación de la AF disponibles para una mejor caracterización de los pacientes con EPOC que nos permita adoptar estrategias terapéuticas acordes a cada paciente.

\section{Evaluación de la actividad física}

La AF se define como "cualquier movimiento corporal producido por los músculos esqueléticos que resulta en un gasto de energía" . Esto incluye tanto las actividades relacionadas con las actividades de la vida diaria (AVD), trabajo o ejercicio físico.

En general, un sujeto puede ser considerado inactivo si no cumple alguno de los siguientes criterios: Al menos 30 min de AF moderada a vigorosa más de 5 días a la semana, al menos $20 \mathrm{~min}$ de actividad vigorosa a intensa 3 veces a la semana, o una combinación de períodos cortos de al menos 10 min de ejercicio moderado (3 veces) o vigoroso (2 veces) a la semana ${ }^{10}$.

La evaluación de la AF es una de las variables relevantes que se deben considerar tanto en per- sonas sanas como en personas con enfermedades, ya que permite establecer cambios posteriores a una intervención.

\section{Sistemas de medición de la actividad física}

La AF puede ser evaluada de diferentes formas. A continuación, presentamos los métodos de evaluación disponibles (Figura 1, Tabla 1):

\section{Cuestionarios}

La evaluación subjetiva de la AF en pacientes con EPOC se puede estimar con cuestionarios que son fáciles y económicos de utilizar, motivo por el cual, son herramientas de preferencia en estudios epidemiológicos ${ }^{11}$. Existen una variedad de cuestionarios que permiten evaluar diferentes aspectos de la AF como: cantidad, tipo, intensidad, sintomatología y limitaciones en las $\mathrm{AVD}^{11}$. Estas herramientas utilizan, para extrapolar el gasto energético realizado, el tiempo y la intensidad de la AF identificadas por las personas. Pueden ser cuestionarios autoadministrados, que son completados por el paciente o guiados por profesionales capacitados. Lo importante es que estos instrumentos sean específicos y validadas sus propiedades psicométricas ${ }^{12}$.

Una limitación importante es que sólo evalúan las actividades más comunes, dejando fuera actividades poco frecuentes, pero que pueden involucrar un gran gasto energético.

\section{Evaluación Actividad Física}

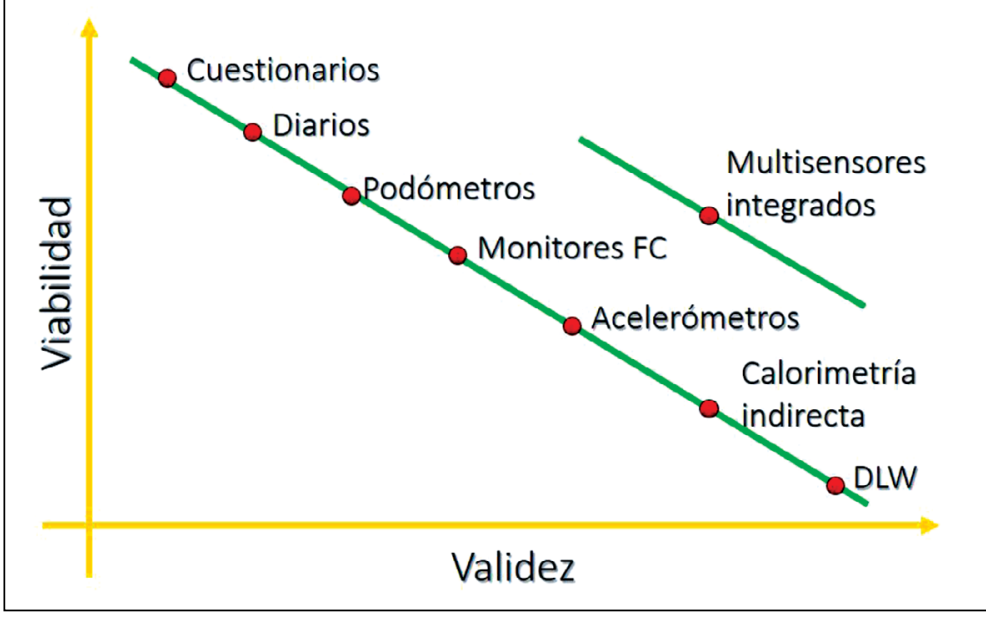

Figura 1. Representación esquemática de las distintas herramientas para evaluar la actividad física según validez y viabilidad. Autorizado por Garcia-Aymerich et al. 
Tabla 1. Comparación de los diferentes métodos de evaluación

\begin{tabular}{|c|c|c|c|}
\hline $\begin{array}{l}\text { Dispositivo/ } \\
\text { instrumento }\end{array}$ & Parámetros evaluados & Ventajas & Desventajas \\
\hline Cuestionarios & $\begin{array}{l}\text { Síntomas limitantes } \\
\text { Tipo e intensidad del ejercicio } \\
\text { AVD }\end{array}$ & $\begin{array}{l}\text { Fácil aplicación } \\
\text { Económicos }\end{array}$ & $\begin{array}{l}\text { Medición indirecta } \\
\text { Requieren proceso complejo de } \\
\text { validación } \\
\text { Utilizan datos subjetivos }\end{array}$ \\
\hline Diarios & $\begin{array}{l}\text { Síntomas limitantes } \\
\text { AVD } \\
\text { Tipo e intensidad del ejercicio }\end{array}$ & $\begin{array}{l}\text { Fácil aplicación } \\
\text { Económicos }\end{array}$ & $\begin{array}{l}\text { Medición indirecta } \\
\text { Requieren proceso complejo de } \\
\text { validación } \\
\text { Utiliza datos subjetivos } \\
\text { Baja confiabilidad }\end{array}$ \\
\hline Podómetros & $\begin{array}{l}\text { Números de pasos } \\
\text { Gasto energético } \\
\text { Distancia caminada }\end{array}$ & $\begin{array}{l}\text { Pequeños } \\
\text { Livianos } \\
\text { Evalúa parámetro objetivo } \\
\text { Portables }\end{array}$ & $\begin{array}{l}\text { Puede subestimar número de } \\
\text { pasos y gasto energético en } \\
\text { marcha a baja velocidad }\end{array}$ \\
\hline Acelerómetros & $\begin{array}{l}\text { Aceleración producida por el } \\
\text { movimiento del individuo en } \\
\text { distintos ejes } \\
\text { Número de pasos } \\
\text { Tiempo utilizado en tipo de } \\
\text { actividad } \\
\text { Intensidad de la actividad }\end{array}$ & $\begin{array}{l}\text { Liviano } \\
\text { Fácil de utilizar } \\
\text { Evalúa parámetro objetivo } \\
\text { Posible combinar con sensores } \\
\text { fisiológicos de FC, temperatura } \\
\text { de la piel } \\
\text { Validados }\end{array}$ & $\begin{array}{l}\text { Baja utilidad en personas con } \\
\text { limitación severa de funciona- } \\
\text { lidad }\end{array}$ \\
\hline $\begin{array}{l}\text { Calorimetría } \\
\text { Indirecta }\end{array}$ & $\begin{array}{l}\text { Gasto energético evalúa relación } \\
\text { existente entre consumo de } \\
\text { oxígeno y producción de dióxido } \\
\text { de carbono }\end{array}$ & $\begin{array}{l}\text { Medición objetiva y confiable de } \\
\text { gasto energético }\end{array}$ & $\begin{array}{l}\text { Baja aplicación clínica } \\
\text { Elevado costo } \\
\text { Procedimiento complejo }\end{array}$ \\
\hline Agua DM & Gasto energético & Medición objetiva & $\begin{array}{l}\text { Validada solo en personas sanas } \\
\text { No diferencia distintos tipos e } \\
\text { intensidad de actividad física }\end{array}$ \\
\hline
\end{tabular}

AVD: Actividades de la vida diaria; FC: Frecuencia cardiaca; Agua DM: Agua doblemente marcada.

En una revisión realizada por Frei, se evaluó la totalidad de los cuestionarios disponibles para medir la AF en adultos mayores y pacientes con enfermedad crónica. De un total de 104 cuestionarios identificados, 15 fueron desarrollados para ser utilizados en pacientes con EPOC. En este estudio, solo $85 \%$ de los cuestionarios estaban correctamente validados, motivo por el cual es importante constatar su validación al momento de aplicarlos ${ }^{13}$.

Entre los cuestionarios disponibles, destaca el International Physical Activity Questionnaire (IPAQ), que ha sido validado en varios idiomas, incluido el español ${ }^{14}$. En nuestro país, Parada y cols, utilizando el IPAQ, encontraron que en pacientes con EPOC, la actividad física está reducida en magnitud e intensidad, especialmente en EPOC moderada y cuando ya existe disnea leve comparada con controles sanos ${ }^{15}$. Otro instrumento valida- do y ampliamente utilizado es el Cuestionario de Actividad Física Global (GPAQ) que es el que ha permitido conocer los niveles de actividad física de la población chilena, debido a que es el utilizado en la Encuesta Nacional de Salud ${ }^{16}$.

\section{Diarios}

Los diarios son otra herramienta de evaluación subjetiva de la AF. En los diarios, se estima el gasto energético en relación a la actividad realizada y al tiempo de duración. Los diarios pueden proveer información muy detallada de los tipos de actividad y sus patrones (por ej. caminar, ver televisión), propósito (por ej. ejercicio, ocupacional, transporte, etc.), intensidad (leve, moderada, vigorosa), duración (en minutos u horas), frecuencia y posición corporal (por ej. sentado, de pie, caminando $)^{17}$.

Uno de los más utilizados es el diario de Fo- 
llick, que se creó para evaluar dolor en pacientes con patologías crónicas y que en investigaciones posteriores ha sido utilizado en estudios comparativos de distintas formas de evaluación de la $\mathrm{AF}$ en pacientes con $\mathrm{EPOC}^{18}$. Han demostrado una buena correlación con las exacerbaciones ${ }^{19}$.

Una de sus principales desventajas es que el compromiso cognitivo reportado en pacientes con EPOC $^{20}$ puede afectar su ejecución. En general, la fiabilidad de estas evaluaciones es baja.

\section{Podómetros}

Los podómetros son dispositivos portables, pequeños, livianos y no invasivos que cuentan el número de pasos que un sujeto realiza en una determinada cantidad de tiempo ${ }^{21}$. Algunos dispositivos, a partir del número de pasos, pueden estimar la distancia y el gasto energético en que se ha incurrido ${ }^{11}$. Existe una gran variedad y son muy sensibles en contar los pasos, pero su confiabilidad disminuye al estimar la distancia y el gasto energético ${ }^{22}$. Uno de los problemas que presentan es que pueden subestimar el número de pasos y el gasto energético cuando se realiza caminata a baja velocidad, como la realizada por pacientes en estadios avanzados de la enfermedad ${ }^{23}$.

Estos dispositivos pueden proveer una retroalimentación a los pacientes respecto de sus AVD y han sido recomendados como una herramienta útil para aumentar los niveles de actividad ${ }^{12}$. En cuanto a los pacientes con EPOC, Mendoza y cols mostraron que en un programa de tres meses de duración, que el uso de podómetros mejoró la AF en 3.080 pasos promedio, además de mejorar la calidad de vida y la capacidad física evaluada a través del test de marcha de $6 \mathrm{~min}(\mathrm{TM} 6 \mathrm{M})^{24}$. Actualmente se han entregado recomendaciones de AF a partir del número de pasos por día, utilizándose 10.000 pasos diarios como recomendación ${ }^{25}$, principalmente porque se han demostrado efectos beneficiosos para la salud y por ser un número fácil de recordar.

Un estudio preliminar, realizado en 74 pacientes con EPOC, sugiere que un cambio clínicamente significativo después de un programa de rehabilitación debería variar entre 600 y 1.100 pasos por día respecto a los valores basales ${ }^{26}$.

Si bien los podómetros no son buenos estimadores de actividad física, sí se recomienda su uso en los programas que incentivan la realización de esta, debido a que entregan una retroalimentación al paciente, permiten plantear metas, son de bajo costo y son fáciles de utilizar.

\section{Acelerómetros}

Los acelerómetros son dispositivos electrónicos portátiles que detectan la aceleración y de esta forma reflejan el movimiento corporal ${ }^{21}$. Se utilizan para cuantificar la actividad realizada $y$, además, mediante el uso de algoritmos, entregar información acerca del tiempo utilizado en los diferentes niveles de intensidad de la AF, así como el número de pasos y una estimación del gasto energético ${ }^{11}$.

El uso de estos dispositivos entregan una información objetiva que no se puede obtener a través de cuestionarios o podómetros ${ }^{21}$. Existe una gran diversidad de dispositivos que pueden detectar movimientos en uno, dos o tres ejes.

Los acelerómetros uniaxiales entregan información similar a los podómetros, pero con la ventaja que pueden medir la aceleración. Como la gran cantidad de movimientos que realizan las personas son en más de un plano, se prefiere utilizar acelerómetros bi o triaxiales ${ }^{6}$. En algunos casos, los acelerómetros vienen combinados con otros sensores fisiológicos, como la frecuencia cardiaca (FC) o la temperatura de la piel, y a partir de esta combinación aumenta su precisión para estimar la AF diaria y el gasto energético ${ }^{27}$.

El tiempo de medición de la AF es un factor importante que puede influir en la interpretación de los datos. En los pacientes graves con 2 o 3 días es suficiente, sin embargo, en los pacientes leves se debe considerar sobre 5 días $^{7}$. Para determinar cambios en los niveles de AF posterior a un programa de rehabilitación son suficientes cuatro días de semana, incluyendo como mínimo $8 \mathrm{~h}$ diarias, considerando las horas de luz durante el día ${ }^{28}$. Es importante al momento del análisis comparar días de semana con los de fin de semana de manera separada.

Debido a la gran variedad de dispositivos utilizados, han aparecido en la literatura investigaciones que los comparan. Van Remoortel ${ }^{29}$ comparó 6 acelerómetros, determinando que el DynaPort MiniMod (McRoberts BV, La Haya, Holanda), el Actigraph GT3X (Actigraph, Pensacola, EEUU) y el SenseWear Armband (BodyMedia Inc, Pittsburgh, PA, EEUU) fueron los más válidos y sensibles para medir AF en pacientes con EPOC. Este tipo de dispositivos tienen un costo elevado y para poder 
utilizarlos se requiere un software específico para cada modelo, en el caso del Dynaport Minimod, cada equipo se debe conectar a Internet y se envía el archivo de registro a Holanda, y al cabo de unos minutos se recibe el análisis por el mismo medio. El costo del equipo más el software es cercano a los 1.000 euros y se debe pagar un costo pequeño de renovación cada año. Actigraph tiene un costo un poco menor y también debe renovarse la licencia periódicamente. Ambos equipos se comercializan en Chile. Por último, SenseWear Armband no está disponible en la actualidad en nuestro país.

Además, cabe destacar que existen en el mercado acelerómetros de bajo costo, sin embargo, para poder utilizarlos se debe lograr descargar la información e interpretarla a través de softwares que requieren conocimientos en programación.

Por último, es importante recalcar que los acelerómetros presentan ciertas limitaciones, especialmente en sujetos con alteraciones funcionales importantes en la marcha. Además, en las AVD la medición de la AF puede ser inadecuada ${ }^{30}$. Por otro lado, la vibración de los vehículos puede sobreestimar la AF, aunque este factor puede ser minimizado filtrando la señal del acelerómetro ${ }^{31}$.

\section{Otros métodos}

Existen otros métodos poco utilizados, principalmente por su costo y la disponibilidad en los centros de salud, entre ellos tenemos la calorimetría indirecta y el agua doblemente marcada.

La calorimetría indirecta $(\mathrm{CI})$ es una técnica utilizada para determinar el gasto energético basal (GEB) de una persona. Estima el gasto energético midiendo el intercambio gaseoso en base a la relación entre el consumo de oxígeno $\left(\mathrm{O}_{2}\right)$, la producción de dióxido de carbono $\left(\mathrm{CO}_{2}\right)$ y la tasa de oxidación de los sustratos. Este enfoque se basa en la relación entre el consumo de oxígeno y la energía producida, por ejemplo, por cada litro de oxígeno consumido por el cuerpo, se utilizan aproximadamente $5 \mathrm{kcal}$. En términos simples, a través de la medición del consumo de oxígeno durante tareas como descansar, estar de pie, caminar o correr, se puede determinar el costo energético de esa actividad ${ }^{32}$. Esta técnica puede ser utilizada para medir el metabolismo basal o gasto energético en reposo, así como también el gasto producido por una determinada $\mathrm{AF}$, el efecto termogénico de una dieta y el gasto energético total ${ }^{33}$. Este método es el más común para cuantificar el gasto energético en mediciones de laboratorio.

La técnica del agua doblemente marcada es el gold standard para la medición del gasto energético $^{34}$. Esta evaluación provee una medición indirecta del gasto energético total durante un período prolongado (por ejemplo 2 semanas). Consiste en la ingesta de dosis de deuterio y oxígeno $18^{35}$. El deuterio es eliminado a través de la orina y el oxígeno 18 es eliminado como agua de orina y $\mathrm{CO}_{2}$. La diferencia entre el lavado de los dos provee una estimación de producción de $\mathrm{CO}_{2}$ que se extrapola a gasto energético ${ }^{36}$. Aproximadamente de 5 a 20\% de estos trazadores se pierde diariamente. Una de sus ventajas es que no es necesario medir el $\mathrm{CO}_{2}$ espirado, sino simplemente recolectar periódicamente una muestra de orina, saliva o plasma para determinar la cantidad de isótopos que se eliminan. Una de sus principales desventajas es que no separa el gasto energético asociado de la AF del asociado a su tasa metabólica basal. Por lo tanto, el valor obtenido es el promedio del gasto energético, pero no cuantifica actividad, tipo, intensidad o duración de este gasto energético ${ }^{37}$. Se ha utilizado en estudios en pacientes con $\mathrm{EPOC}^{38}$, sin embargo, presenta algunas limitaciones debido a que el método asume los niveles de AF a partir de sujetos sanos y no con enfermedad.

\section{Efectos de la inactividad física en los pacientes con EPOC}

La inactividad física es una causa o consecuencia común de muchas enfermedades crónicas. La evidencia ha mostrado que la AF reducida predispone a enfermedades cardiovasculares ${ }^{39}$, obesidad $^{40} \mathrm{y}$ diabetes mellitus ${ }^{41}$ entre otras enfermedades crónicas no transmisibles.

Las personas con EPOC presentan una menor cantidad de AF, y esta condición se ve exacerbada a medida que se avanza en el estadio de la enfermedad $^{7}$. La causa de la disminución de la AF está asociada al aumento de la disnea y esto tiene un componente multifactorial.

La literatura actual sugiere que los niveles de AF se reducen tempranamente en el desarrollo de la EPOC ${ }^{42}$. En un estudio que evaluó 177 pacientes con EPOC con un volumen espiratorio forzado al primer segundo $\left(\mathrm{VEF}_{1}\right)$ promedio de $52 \%$ del 
teórico, sólo 1 de cada 4 sujetos alcanzó como mínimo 30 min consecutivos de AF moderada a vigorosa ${ }^{43}$. Comparado con controles, los pacientes con EPOC han mostrado una reducción de $50 \%$ del tiempo de AF moderada a vigorosa, medida en minutos, por día ${ }^{44}$.

La literatura reporta que realizar AF de manera regular está relacionado con disminución en la mortalidad por causa respiratoria y por todas las causas. Tres estudios longitudinales, con un seguimiento de 3 a 12, años mostraron que bajos niveles de AF predicen mortalidad por todas las causas en pacientes con EPOC ${ }^{45-47}$. La relación fue consistente a pesar de las diferencias entre los pacientes evaluados y los métodos de evaluación. También, bajos niveles de AF han sido asociados con alto riesgo de hospitalización y rehospitalización $^{46,48}$.

El nivel de AF es un factor importante en determinar la calidad de vida y el estado de salud, reportándose que pacientes físicamente más activos tienen mejores niveles en cuestionarios de calidad de vida relacionada a la salud ${ }^{49}$.

Los principales factores que se asocian con la $\mathrm{AF}$ en los pacientes con EPOC son:

\section{Función pulmonar}

El $\mathrm{VEF}_{1}$ y la capacidad de difusión han mostrado una asociación débil a moderada con los niveles de AF en pacientes con $\mathrm{EPOC}^{50}$. En cambio, la hiperinsuflación dinámica, si ha mostrado una asociación robusta con el nivel de AF, probablemente debido a su importancia en la génesis de la disnea ${ }^{51}$.

\section{Capacidad física}

Se ha encontrado una asociación moderada entre la capacidad de realizar AF con el TM6M y con la carga máxima de trabajo en una prueba incremental ${ }^{7}$.

\section{Factores sociodemográficosy medioambientales}

La etnia, status socioeconómico, disponibilidad de trabajo, nivel educacional, el clima, condiciones de altitud sobre el nivel del mar, pueden influenciar la realización de AF entre personas con $\mathrm{EPOC}^{21}$. Algunos factores como temperaturas extremas o contaminantes ambientales, que incrementan los síntomas, pueden transformarse en barreras y disminuir la AF en estos sujetos ${ }^{52}$.

\section{Exacerbaciones}

La AF está reducida en gran magnitud durante y después de una hospitalización ${ }^{53}$, e independiente del tiempo de recuperación, los niveles previos de AF podrían no ser recuperados. La literatura ha mostrado que sujetos con más de una exacerbación tienen menores niveles de $\mathrm{AF}^{52}$.

Además, se ha reportado que influyen las comorbilidades, la inflamación sistémica, el estado de salud, la presencia de síntomas y la autoconvicción ${ }^{52}$.

\section{Conclusión}

La AF está disminuida en los pacientes con EPOC y está asociada a una mayor mortalidad y probabilidad de hospitalización. Debido a esto, es imprescindible evaluar la AF de estos sujetos, y así determinar mejor el efecto de nuestras intervenciones, en especial en sujetos que consulten por disnea y menor tolerancia al ejercicio y en aquellos que ingresarán a un programa de rehabilitación

Sabemos que la inactividad física es un factor de riesgo modificable, en que la intervención no solo contribuirá a disminuir la probabilidad de mortalidad, sino que también disminuirá las comorbilidades, que juegan un rol principal en la limitación funcional y la alteración en la participación de estos sujetos, lo que redundará en una mejor calidad de vida.

Es por lo anterior que es necesario contar con herramientas objetivas que permitan una evaluación fidedigna de la AF que realizan estos pacientes. Además, las estrategias para evaluar la AF deben ser sensibles para detectar cambios producidos por la evolución de la propia enfermedad, de las intervenciones terapéuticas administradas, permitiendo cubrir distintos rangos de severidad y ser adaptables a las limitaciones físicas o psicológicas.

Nuestra recomendación es utilizar los acelerómetros disponibles para una valoración objetiva de la actividad física, pero, si no se cuenta con recursos, utilizar los cuestionarios IPAQ y GPAQ que han sido validados en nuestro idioma, son fáciles de utilizar y cuentan con respaldo en la literatura científica nacional y en evaluaciones tan relevantes como la Encuesta Nacional de Salud.

Si bien la evaluación de la actividad física es recomendable en todo paciente con enfermedad 
respiratoria crónica, la recomendación en la literatura de estos dispositivos para fomentar la actividad física es más cautelosa, por lo que se debe seguir investigando en esta área.

Por último, es necesario conocer las ventajas y desventajas de las múltiples herramientas de evaluación de la AF disponibles, para así elegir la mejor opción en un contexto determinado por las circunstancias individuales de cada paciente.

\section{Referencias}

1. Torres-Castro R, Zenteno D, Rodríguez-Núñez I, Villarroel G, Alvarez C, Gatica D, et al. Guías de rehabilitación respiratoria en niños con enfermedades respiratorias crónicas: actualización 2016. Neumol Pediatr. 2016; 11 (3): 114-31.

2. Vestbo J, Hurd SS, Agusti AG, Jones PW, Vogelmeier C, Anzueto A, et al. Global strategy for the diagnosis, management, and prevention of chronic obstructive pulmonary disease: GOLD executive summary. Am J Respir Crit Care Med 2013; 187 (4): 347-65.

3. Murray CJ, Lopez AD. Alternative projections of mortality and disability by cause 1990-2020: Global Burden of Disease Study. The Lancet 1997; 349 (9064): 1498-504.

4. Menezes AMB, Perez-Padilla R, Jardim JB, Muiño A, Lopez MV, Valdivia G, et al. Chronic obstructive pulmonary disease in five Latin American cities (the PLATINO study): a prevalence study. The Lancet 2005; 366 (9500): 1875-81.

5. Cooper CB. Airflow obstruction and exercise. Respir Med 2009; 103 (3): 325-34.

6. Troosters T, van der Molen T, Polkey M, Rabinovich RA, Vogiatzis I, Weisman I, et al. Improving physical activity in COPD: towards a new paradigm. Respir Res 2013; 14 (1): 115.

7. Watz H, Waschki B, Meyer T, Magnussen H. Physical activity in patients with COPD. Eur Respir J 2009; 33 (2): 262-72.

8. Garcia-Aymerich J, Farrero E, Felez M, Izquierdo J, Marrades R, Anto J. Risk factors of readmission to hospital for a COPD exacerbation: a prospective study. Thorax 2003; 58 (2): 100-5.

9. Caspersen CJ, Powell KE, Christenson GM. Physical activity, exercise, and physical fitness: definitions and distinctions for health-related research. Public Health Rep 1985; 100 (2): 126.

10. Haskell WL, Lee I-M, Pate RR, Powell KE, Blair SN, Franklin BA, et al. Physical activity and public health: updated recommendation for adults from the American College of Sports Medicine and the American Heart
Association. Circulation 2007; 116 (9): 1081.

11. Pitta F, Troosters T, Probst V, Spruit M, Decramer M, Gosselink R. Quantifying physical activity in daily life with questionnaires and motion sensors in COPD. Eur Respir J 2006; 27 (5): 1040-55.

12. Terwee CB, Mokkink LB, van Poppel MN, Chinapaw MJ, van Mechelen W, de Vet HC. Qualitative attributes and measurement properties of physical activity questionnaires. Sports Med 2010; 40 (7): 525-37.

13. Frei A, Williams K, Vetsch A, Dobbels F, Jacobs L, Rüdell $\mathrm{K}$, et al. A comprehensive systematic review of the development process of 104 patient-reported outcomes (PROs) for physical activity in chronically ill and elderly people. Health Qual Life Outcomes 2011; 9 (1): 116.

14. Craig CL, Marshall AL, Sjöström M, Bauman AE, Booth $\mathrm{ML}$, Ainsworth BE, et al. International physical activity questionnaire: 12 -country reliability and validity. Med Sci Sports Exerc 2003; 35 (8): 1381-95.

15. Parada A, Klaassen J, Lisboa C, Saldías F, Mendoza L, Díaz O. Reducción de la actividad física en pacientes con enfermedad pulmonar obstructiva crónica. Rev Med Chile 2011; 139 (12): 1562-72.

16. Ministerio de Salud de Chile. Encuesta Nacional de Salud 2009-2010. Disponible en: http://web.minsal.cl/ portal/url/item/bcb03d7bc28b64dfe040010165012d23. pdf (Citado el 26 de diciembre de 2016).

17. Ainsworth BE. How do I measure physical activity in my patients? Questionnaires and objective methods. $\mathrm{Br}$ J Sports Med 2009; 43 (1): 6-9.

18. Moore R, Berlowitz D, Denehy L, Jackson B, McDonald CF. Comparison of pedometer and activity diary for measurement of physical activity in chronic obstructive pulmonary disease. J Cardiopulm Rehabil Prev 2009; 29 (1): 57-61.

19. Johnston NW, Lambert K, Hussack P, de Verdier MG, Higenbottam T, Lewis J, et al. Detection of COPD Exacerbations and Compliance With Patient-Reported Daily Symptom Diaries Using a Smartphone-Based Information System. Chest 2013; 144 (2): 507-14.

20. Incalzi RA, Marra C, Giordano A, Calcagni ML, Cappa A, Basso S, et al. Cognitive impairment in chronic obstructive pulmonary disease. J Neurol 2003; 250 (3): 325-32.

21. Watz H, Pitta F, Rochester CL, Garcia-Aymerich J, ZuWallack R, Troosters T, et al. An official European Respiratory Society statement on physical activity in COPD. Eur Respir J 2014; 44 (6): 1521-37.

22. Schneider PL, Crouter SE, Lukajic O, Bassett DR. Accuracy and reliability of 10 pedometers for measuring steps over a 400-m walk. Med Sci Sports Exerc 2003; 35 (10): 1779-84. 
23. Turner LJ, Houchen L, Williams J, Singh SJ. Reliability of pedometers to measure step counts in patients with chronic respiratory disease. J Cardiopulm Rehabil Prev 2012; 32 (5): 284-91.

24. Mendoza L, Horta P, Espinoza J, Aguilera M, Balmaceda $\mathrm{N}$, Castro A, et al. Pedometers to enhance physical activity in COPD: a randomised controlled trial. Eur Respir J 2015; 45 (2): 347-54.

25. Tudor-Locke C, Craig CL, Aoyagi Y, Bell RC, Croteau KA, De Bourdeaudhuij I, et al. How many steps/day are enough? For older adults and special populations. Int J Behav Nutr Phys Act 2011; 8 (1): 80

26. Demeyer H, Burtin C, Hornikx M, Camillo CA, Van Remoortel H, Langer D, et al. The Minimal Important Difference in Physical Activity in Patients with COPD. PloS One 2016; 11 (4): e0154587.

27. Patel SA, Benzo RP, Slivka WA, Sciurba FC. Activity monitoring and energy expenditure in COPD patients: a validation study. COPD 2007; 4 (2): 107-12.

28. Demeyer H, Burtin C, Van Remoortel H, Hornikx M, Langer D, Decramer M, et al. Standardizing the analysis of physical activity in patients with COPD following a pulmonary rehabilitation program. Chest 2014; 146 (2): 318-27.

29. Van Remoortel H, Raste Y, Louvaris Z, Giavedoni S, Burtin C, Langer D, et al. Validity of six activity monitors in chronic obstructive pulmonary disease: a comparison with indirect calorimetry. PloS One 2012; 7 (6): e39198.

30. Strath SJ, Pfeiffer KA, Whitt-Glover MC. Accelerometer use with children, older adults, and adults with functional limitations. Med SciSports Exerc 2012; 44 (1 Suppl 1): S77.

31. Cohen MD, Cutaia M, Brehm R, Brutus V, Courtney Pike V, Lewendowski D. Detecting motor vehicle travel in accelerometer data. COPD 2012; 9 (2): 102-10.

32. Hills AP, Mokhtar N, Byrne NM. Assessment of physical activity and energy expenditure: an overview of objective measures. Front Nutr 2014; 1 (5): 1-16.

33. López-Fontana C, Martínez-González M, Martínez J. Obesidad, metabolismo energético y medida de la actividad física. Rev Esp Obes 2003; 1 (1): 29-36.

34. Schoeller D, Van Santen E. Measurement of energy expenditure in humans by doubly labeled water method. J Appl Physiol 1982; 53 (4): 955-9.

35. Westerterp K, Wouters L, van Marken LW. The Maastricht protocol for the measurement of body composition and energy expenditure with labeled water. Obes Res 1995; 3: 49-57.

36. Schoeller DA. Recent advances from application of doubly labeled water to measurement of human energy expenditure. J Nutr 1999; 129 (10): 1765-8.

37. DeLany JP, Lovejoy JC. Energy expenditure. Endocrinol Metab Clin North Am 1996; 25 (4): 831-46.

38. Baarends EM, Schols A, Pannemans D, Westerterp $\mathrm{KR}$, Wouters E. Total free living energy expenditure in patients with severe chronic obstructive pulmonary disease. Am J Respir Crit Care Med 1997; 155 (2): 549-54.

39. Mora S, Cook N, Buring JE, Ridker PM, Lee I-M. Physical activity and reduced risk of cardiovascular events potential mediating mechanisms.Circulation 2007; 116 (19): 2110-8.

40. Li S, Zhao JH, Luan Ja, Ekelund U, Luben RN, Khaw $\mathrm{K}-\mathrm{T}$, et al. Physical activity attenuates the genetic predisposition to obesity in 20,000 men and women from EPIC-Norfolk prospective population study.PLoS Med 2010; 7 (8): 1064.

41. Jefferis BJ, Whincup PH, Lennon L, Wannamethee SG. Longitudinal Associations Between Changes in Physical Activity and Onset of Type 2 Diabetes in Older British Men The influence of adiposity. Diabetes Care 2012; 35 (9): 1876-83.

42. Van Remoortel H, Hornikx M, Demeyer H, Langer D, Burtin C, Decramer M, et al. Daily physical activity in subjects with newly diagnosed COPD. Thorax 2013; 68 (10): 962-3.

43. Donaire-González D, Gimeno-Santos E, Balcells E, Rodríguez DA, Farrero E, de Batlle J, et al. Physical activity in COPD patients: patterns and bouts. Eur Respir J 2013; 42 (4): 993-1002.

44. Van Remoortel H, Camillo CA, Langer D, Hornikx M, Demeyer H, Burtin C, et al. Moderate intense physical activity depends on selected Metabolic Equivalent of Task (MET) cut-off and type of data analysis. PloS One 2013; 8 (12): e84365.

45. Waschki B, Kirsten A, Holz O, Müller K-C, Meyer T, Watz $\mathrm{H}$, et al. Physical activity is the strongest predictor of all-cause mortality in patients with COPD: a prospective cohort study. Chest 2011; 140 (2): 331-42.

46. García-Río F, Rojo B, Casitas R, Lores V, Madero R, Romero D, et al. Prognostic value of the objective measurement of daily physical activity in patients with COPD. Chest 2012; 142 (2): 338-46.

47. García-Aymerich J, Lange P, Benet M, Schnohr P, Antó JM. Regular physical activity reduces hospital admission and mortality in chronic obstructive pulmonary disease: a population based cohort study. Thorax 2006; 61 (9): 772-8.

48. Bahadori K, Fitz Gerald JM. Risk factors of hospitalization and readmission of patients with COPD exacerbation-systematic review. Int J Chron Obstruct Pulmon Dis 2007; 2 (3): 241. 
49. Esteban C, Quintana J, Aburto M, Moraza J, Egurrola M, Pérez-Izquierdo J, et al. Impact of changes in physical activity on health-related quality of life among patients with COPD.Eur Respir J 2010; 36 (2): 292300.

50. Langer D, i Iranzo MC, Burtin C, Verleden S, Vanaudenaerde B, Troosters T, et al. Determinants of physical activity in daily life in candidates for lung transplantation. Respir Med 2012; 106 (5): 747-54.

51. Garcia-Rio F, Lores V, Mediano O, Rojo B, Hernanz A, López-Collazo E, et al. Daily physical activity in patients with chronic obstructive pulmonary disease is mainly associated with dynamic hyperinflation. Am J Respir Crit Care Med 2009; 180 (6): 506-12.

52. Waschki B, Spruit MA, Watz H, Albert PS, Shrikrishna $\mathrm{D}$, Groenen $\mathrm{M}$, et al. Physical activity monitoring in COPD: compliance and associations with clinical characteristics in a multicenter study. RespirMed 2012; 106 (4): 522-30.

53. Pitta F, Troosters T, Probst VS, Spruit MA, Decramer M, Gosselink R. Physical activity and hospitalization for exacerbation of COPD. Chest 2006; 129 (3): 536-44. 